\title{
Structural distortions and model Hamiltonian parameters: From LSDA to a tight-binding description of $\mathrm{LaMnO}_{3}$
}

\author{
Claude Ederer, ${ }^{*}$ Chungwei Lin, and Andrew J. Millis \\ Department of Physics, Columbia University, 538 West 120th Street, New York, New York 10027, USA
}

(Received 1 June 2007; published 8 October 2007)

\begin{abstract}
The physics of manganites is often described within an effective two-band tight-binding (TB) model for the Mn $e_{g}$ electrons, which apart from the kinetic energy includes also a local "Hund's rule" coupling to the $t_{2 g}$ core spin and a local coupling to the Jahn-Teller (JT) distortion of the oxygen octahedra. We test the validity of this model by comparing the energy dispersion calculated for the TB model with the full Kohn-Sham band structure calculated within the local spin-density approximation (LSDA) to density functional theory. We analyze the effect of magnetic order, JT distortions, and " $\mathrm{GdFeO}_{3}$-type" tilt rotations of the oxygen octahedra. We show that the hopping amplitudes are independent of magnetic order and JT distortions and that both effects can be described with a consistent set of model parameters if hopping between both nearest and next-nearest neighbors is taken into account. We determine a full set of model parameters from the density functional theory calculations, and we show that both JT distortions and Hund's rule coupling are required to obtain an insulating ground state within LSDA. Furthermore, our calculations show that the $\mathrm{GdFeO}_{3}$-type rotations of the oxygen octahedra lead to a substantial reduction of the hopping amplitudes but to no significant deviation from the simple TB model.
\end{abstract}

DOI: 10.1103/PhysRevB.76.155105

PACS number(s): 75.47.Lx, 71.10.-w

\section{INTRODUCTION}

Manganite systems, $R_{1-x} A_{x} \mathrm{MnO}_{3}$, where $R$ is a trivalent rare earth cation (e.g., $\mathrm{La}^{3+}, \mathrm{Pr}^{3+}, \mathrm{Nd}^{3+}$ etc.) and $A$ is a divalent alkaline earth cation (e.g., $\mathrm{Sr}^{2+}, \mathrm{Ca}^{2+}$, etc.), have attracted the attention of scientists already for decades. ${ }^{1-5}$ These compounds exhibit a very rich phase diagram as a function of both temperature and composition, with various types of eventually coexisting charge, orbital, and magnetic order, and they are therefore important prototype materials to test our current understanding of correlated electron systems. In addition, the observation of "colossal magnetoresistance,"6 a magnetic-field induced change in electric resistivity by several orders of magnitude, has spawned further interest both in the fundamental physics behind this effect as well as in the question of whether this effect can be utilized for technological applications.

$\mathrm{LaMnO}_{3}$, the parent material for many manganite systems, exhibits an orthorhombically distorted perovskite structure with Pnma space group [see Fig. 1(a)]. ${ }^{8}$ The observed deviation from the ideal cubic perovskite structure [shown in Fig. 1(b)] involves both Jahn-Teller (JT) distortions of the oxygen octahedra surrounding the Mn cations ${ }^{9}$ as well as a collective tilting of these octahedra, the so-called " $\mathrm{GdFeO}_{3}$-type" distortion. ${ }^{10}$ The magnetic moments of the Mn cations in $\mathrm{LaMnO}_{3}$ order at $T_{N}=140 \mathrm{~K}$ in a so-called “ $A$-type" antiferromagnetic structure, ${ }^{2}$ with parallel alignment of all moments within a certain (001) plane and antiparallel alignment of the moments between adjacent planes.

In the ideal cubic perovskite structure (see Fig. 1(b)), the $3 d$ states of the Mn cations are split by the crystal field into the lower-lying threefold degenerate $t_{2 g}$ states and the higherlying twofold degenerate $e_{g}$ states. In $\mathrm{LaMnO}_{3}$, the majorityspin $t_{2 g}$ states are fully occupied, whereas the two majorityspin $e_{g}$ levels are filled with only one electron, according to the formal high-spin $d^{4}$ electron configuration of the $\mathrm{Mn}^{3+}$ cation.
The theoretical modeling of manganite systems is usually based on the assumption that the important low energy dynamics of these systems can be described within an effective two-band tight-binding (TB) model for the Mn $e_{g}$ electrons. In this model, electrons can hop between the $e_{g}$ orbitals at neighboring $\mathrm{Mn}$ sites, and the corresponding kinetic energy term in the Hamiltonian is eventually complemented by local terms describing the coupling to the $t_{2 g}$ "core spin," the coupling to the JT distortion of the surrounding oxygen octahedron, and the electron-electron interaction. These models can account for many of the properties observed in manganite systems (see Ref. 5 for a recent review). Reasonable values for the model parameters, which describe the strengths of the various competing interactions, can be inferred from experiments. Nevertheless, it is very desirable to independently calculate values for these parameters based on fundamental "first principles" theory. Evaluating the models using such independently determined parameters provides a stringent test for the accuracy of the model description.

Electronic structure calculations based on density functional theory (DFT) $)^{11,12}$ provide a way to study the ground
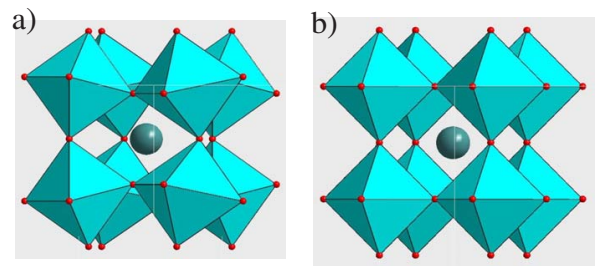

FIG. 1. (Color online) (a) Experimentally observed Pnma structure of $\mathrm{LaMnO}_{3}$ according to Ref. 7. (b) Ideal cubic perovskite structure. The oxygen anions form a network of corner-shared octahedra. The Mn cations (not shown) are situated in the centers of the oxygen octahedra and the La cations occupy the space between the octahedra. 
state electronic structure of a specific material without having to resort to model assumptions and therefore provide a good starting point for the derivation of more simplified models and their parameters (see, e.g., Refs. 13 and 14). The electronic structure of $\mathrm{LaMnO}_{3}$ has been studied previously within the local spin-density approximation (LSDA) to DFT and by using the LSDA $+U$ method. ${ }^{15-18}$ It was shown that many properties such as the correct magnetic ground state and even some spectral properties are well described by these methods, provided that the correct experimental crystal structure is used in the calculation.

Although the model treatment of manganite systems usually employs a pure $e_{g}$ electron description, it is generally understood that the electron hopping between the $e_{g}$ states on neighboring $\mathrm{Mn}$ sites is truly an effective hopping, which is mediated by the intermediate oxygen anions via $d-p$ or $d-s$ hopping. The resulting bands with predominant $e_{g}$ character can be described by an effective two-band model if the Mn $e_{g}$ states are energetically separated from the oxygen $p$ and $s$ states. In this case, the effective nearest-neighbor hopping amplitude $t$ between the $\mathrm{Mn} e_{g}$ states is (to leading order) given by

$$
t \propto \frac{t_{p d}^{2}}{E_{d}-E_{p}} .
$$

Here, $t_{p d}$ is the hopping amplitude between the $\mathrm{Mn} e_{g}$ and the oxygen $p$ states, $E_{d}$ and $E_{p}$ are the energies of the corresponding ionic levels, and for simplicity we have neglected hopping via the oxygen $s$ states.

The JT distortion changes the Mn-O bond lengths, while the octahedral tilts change the bond angles; thus, both distortions affect the overlap integrals which determine the hopping amplitude $t_{p d}$. It is therefore not clear a priori that a simple effective TB model with fixed (distortionindependent) hopping amplitudes $t$ can be used to study the effects of lattice distortions in manganite systems.

Here, we use the Kohn-Sham band structure calculated within the LSDA as a reference for the noninteracting TB model, and we analyze how well the relevant part of the energy dispersion of $\mathrm{LaMnO}_{3}$ can be fitted within an effective two-band TB model for the $e_{g}$ electrons. In particular, we analyze the effects of the two dominant structural distortions in $\mathrm{LaMnO}_{3}$, the JT distortion and the $\mathrm{GdFeO}_{3}$-type rotations, and we address the question of whether magnetic and orbital (JT) orders affect the effective hopping amplitudes.

The result of our analysis is that the effective two-band model gives a good fit of the $e_{g}$-projected Kohn-Sham band structure, provided that hopping between both nearest and next-nearest neighbors is taken into account. We show that the same hopping amplitudes can be used for the ferromagnetic, the A-type antiferromagnetic, and the JT distorted cases, so that the simple two-band TB model can be used to study the effects of JT distortions. Furthermore, we quantify the dependence of the hopping amplitudes on volume changes and on $\mathrm{GdFeO}_{3}$-type rotations. The latter lead to significant reductions of the hopping amplitudes $(\sim 25 \%$ for the experimental structure) relative to the ideal cubic struc- ture with the same unit cell volume. The hopping amplitudes corresponding to the observed bond angles should therefore be used in theoretical modeling.

Our results also provide a quantitative determination of JT and Hund's rule couplings. The result for Hund's coupling is consistent with the previous work; the JT coupling is considerably smaller than previous estimates. ${ }^{19-21}$ We find that both JT and Hund's couplings are required to stabilize the insulating state within LSDA.

Our conclusions rely in an essential way on the energy separation of the transition-metal $d$ bands and the oxygen $p$ bands; methods such as LSDA $+U$ which shift the energy of the transition-metal $d$ bands relative to the energy of the oxygen $p$ bands can produce a band structure that is very poorly described by a simple two-band TB model.

The remaining part of this paper is organized as follows. Section II contains a brief summary of the methods and technical details of our work. We first describe the method we use for our LSDA calculations, then specify the TB Hamiltonian, and finally describe how we decompose the various structural distortions found experimentally in $\mathrm{LaMnO}_{3}$. Our results are discussed in Sec. III, and we end with a summary of our main conclusions and implications for future work.

\section{METHODS AND TECHNICAL DETAILS}

\section{A. Computational method}

We calculate the LSDA Kohn-Sham band structure for $\mathrm{LaMnO}_{3}$ with both ferromagnetic and $A$ type antiferromagnetic orders in various structural modifications using the projector augmented-wave method implemented in the "Vienna ab initio simulation package" (VASP). ${ }^{22-24}$ We treat the La $5 s$, La $5 p$, and Mn $3 p$ pseudocore states as valence states, and we use a plane-wave energy cutoff of $400 \mathrm{eV}$ in all our calculations. We employ $\Gamma$-centered $6 \times 6 \times 6$ and $4 \times 4 \times 3$ $k$-point grids for the calculations corresponding to the simple and quadrupled perovskite unit cells, respectively, and corresponding grids for the structures in which the unit cells are doubled along the $z$ direction or within the $x-y$ plane. These values result in a good convergence of the calculated band structures.

In order to extract the bands resulting from the $\mathrm{Mn} e_{g}$ states, we use the "fatband" technique, i.e., we assign a weight to each calculated eigenvalue, which is proportional to the amount of $\mathrm{Mn} e_{g}$ character contained in the corresponding Bloch function, and we identify the $e_{g}$-derived bands as those containing a non-negligible $e_{g}$ character.

It has been shown in Ref. 15 that the LSDA gives a good overall account of the electronic properties of manganite systems, even though the tendency to open up an energy gap between occupied and unoccupied states is underestimated within the LSDA. This is a well-known feature of the LSDA, which results from the inability of the LSDA to correctly account for the strong Coulomb correlations between the rather localized $d$ states in transition-metal oxides. Such local Coulomb interactions are usually incorporated in the model Hamiltonian via a separate interaction term. In the following, we do not include such an interaction term in our model 


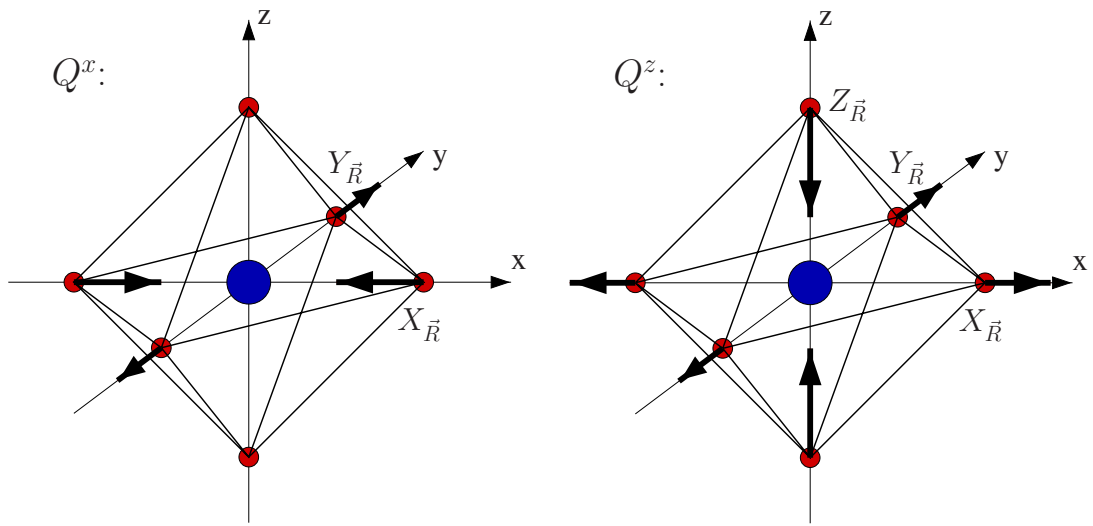

FIG. 2. (Color online) Schematic depiction of the JT modes $Q^{x}$ (left side) and $Q^{z}$ (right side). The displacements $X_{\vec{R}}, Y_{\vec{R}}$, and $Z_{\vec{R}}$ used in Eqs. (6) and (7) are indicated. Since we are considering only inversion symmetric distortions of the oxygen octahedra, it is enough to specify the displacements of the oxygen anions adjacent to the central Mn cation in the positive $x, y$, and $z$ directions. Note that $\left|X_{R}\right|=\left|Y_{R}\right|$ in the case of $Q^{x}$ and $\left|X_{R}\right|=\left|Y_{R}\right|=\frac{1}{2}\left|Z_{\vec{R}}\right|$ for $Q^{z}$.

analysis, and thus the corresponding deficiencies of the LSDA do not affect our results (assuming that the separate treatment of local correlations is justified), except for the question related to the energy separation between the Mn $d$ and the oxygen $p$ states, which is discussed in Sec. IV.

\section{B. Model Hamiltonian}

In Sec. III, we relate the calculated LSDA band structure to the following TB model, which contains the terms that are typically used for the theoretical modeling of manganite systems (see, e.g., Ref. 5):

$$
\hat{H}=\hat{H}_{\text {kin }}+\hat{H}_{\text {Hund }}+\hat{H}_{\mathrm{JT}} \text {, }
$$

with

$$
\begin{gathered}
\hat{H}_{\mathrm{kin}}=-\sum_{\vec{R}, \vec{\delta}, \sigma} \boldsymbol{d}_{\vec{R}, \sigma^{+}}^{+} \boldsymbol{t}_{R, \vec{R}+\vec{\delta}} \boldsymbol{d}_{R+\vec{\delta}, \sigma}, \\
\hat{H}_{\mathrm{Hund}}=-J \sum_{\vec{R}, a, \sigma, \sigma^{\prime}} \vec{S}_{\vec{R}} \cdot \vec{\tau}_{\sigma, \sigma^{\prime}} d_{\vec{R}, a, \sigma}^{+} d_{\vec{R}, a, \sigma^{\prime}}, \\
\hat{H}_{\mathrm{JT}}=-\lambda \sum_{\vec{R}, \sigma}\left(Q_{\vec{R}}^{x} \boldsymbol{d}_{\vec{R}, \sigma}^{+} \boldsymbol{\tau}^{x} \boldsymbol{d}_{R, \sigma}+Q_{\vec{R}}^{z} \boldsymbol{d}_{\vec{R}, \sigma}^{+} \boldsymbol{\tau}^{z} \boldsymbol{d}_{R, \sigma}\right) .
\end{gathered}
$$

Here, $d_{R, a, \sigma}$ is the annihilation operator for an $e_{g}$ electron at site $\vec{R}$ in orbital $a$ with spin $\sigma$, and the corresponding boldface symbol indicates a pseudospinor in orbital space $\boldsymbol{d}_{R, \sigma}$ $=\left(d_{R, 1, \sigma}, d_{R, 2, \sigma}\right)^{T}$. The orbital indices 1 and 2 correspond to $\left|3 z^{2}-r^{2}\right\rangle$ and $\left|x^{2}-y^{2}\right\rangle$ orbitals, respectively. $\boldsymbol{t}_{R}, \vec{R}+\vec{\delta}$ $=\sum_{i=0}^{3} t_{R, \vec{R}+\vec{\delta}}^{i} \vec{\tau}^{i}$ are the hopping amplitudes between site $\vec{R}$ and $\vec{R}+\vec{\delta}$ and $\tau^{i}$ are the usual Pauli matrices supplemented by the $2 \times 2$ unit matrix. $\vec{S}_{R}$ is the normalized core spin of the $t_{2 g}$ electrons $\left(\left|\vec{S}_{\vec{R}}\right|=1\right)$ and $Q_{\vec{R}}^{x, z}$ are the amplitudes of the two JT modes at site $\vec{R}$ that couple to the $e_{g}$ electrons,

$$
Q_{\vec{R}}^{x}=\frac{1}{\sqrt{2}}\left(X_{R}-Y_{R}\right),
$$

$$
Q_{\vec{R}}^{z}=\frac{1}{\sqrt{6}}\left(2 Z_{R}-X_{R}-Y_{R}\right)
$$

Here, $X_{R}, Y_{R}$, and $Z_{\vec{R}}$ are the displacements along $\hat{x}, \hat{y}$, and $\hat{z}$ of the oxygen anions that are situated adjacent to the Mn site at $\vec{R}$ in $x, y$, and $z$ directions, respectively, and only inversion symmetric distortions of the oxygen octahedra are taken into account (see Fig. 2). $J$ and $\lambda$ are coupling constants for the local interaction terms.

The first term in Eq. (2) describes the hopping between neighboring Mn sites. We will consider hopping between both nearest and next-nearest neighbors. Symmetry dictates that the hopping matrices for the nearest-neighbor hopping are

$$
\begin{gathered}
\boldsymbol{t}_{R, R \pm a \hat{x}}=\frac{t}{4}\left(\begin{array}{cc}
1 & -\sqrt{3} \\
-\sqrt{3} & 3
\end{array}\right), \\
\boldsymbol{t}_{R, R \pm a \hat{y}}=\frac{t}{4}\left(\begin{array}{cc}
1 & \sqrt{3} \\
\sqrt{3} & 3
\end{array}\right), \\
\boldsymbol{t}_{R, R \pm a \hat{z}}=t\left(\begin{array}{cc}
1 & 0 \\
0 & 0
\end{array}\right),
\end{gathered}
$$

and for the next-nearest-neighbor hopping,

$$
\begin{gathered}
\boldsymbol{t}_{R, \vec{R} \pm a \hat{x} \pm a \hat{z}}=\frac{t^{\prime}}{2}\left(\begin{array}{cc}
-2 & \sqrt{3} \\
\sqrt{3} & 0
\end{array}\right), \\
\boldsymbol{t}_{R, \vec{R} \pm a \hat{y} \pm a \hat{z}}=\frac{t^{\prime}}{2}\left(\begin{array}{cc}
-2 & -\sqrt{3} \\
-\sqrt{3} & 0
\end{array}\right), \\
\boldsymbol{t}_{R, \vec{R} \pm a \hat{x} \pm a \hat{y}}=\frac{t^{\prime}}{2}\left(\begin{array}{cc}
1 & 0 \\
0 & -3
\end{array}\right) .
\end{gathered}
$$

Here, $a$ is the lattice constant of the underlying cubic perovskite lattice.

The second and third terms in Eq. (2) describe Hund's rule coupling to the $t_{2 g}$ core spin and the coupling to the JT distortion of the oxygen octahedra surrounding site $\vec{R}$, respectively. The normalized $t_{2 g}$ core spin is treated as a classical vector which is fixed externally in our model calculations. The values of $Q_{\vec{R}}^{x / z}$ are given by the positions of the 

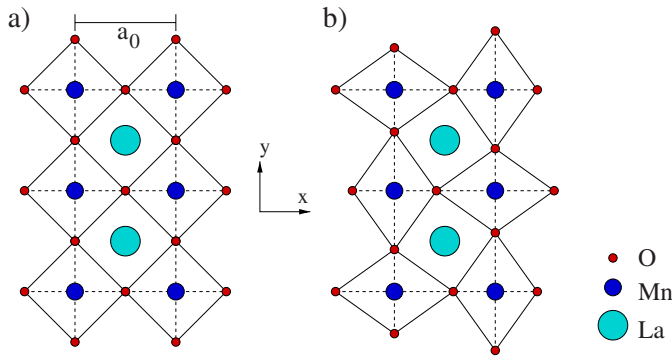

FIG. 3. (Color online) Staggered $Q^{x}$-type JT distortion within the $x-y$ plane found experimentally in $\mathrm{LaMnO}_{3}$. The long and short axes of the octahedra alternate along the $x$ and $y$ directions, as shown in (b), compared to the ideal structure shown in (a).

oxygen anions used in our LSDA calculations and are also treated as external parameters in the TB model.

\section{Structural decomposition}

As described in the Introduction, $\mathrm{LaMnO}_{3}$ exhibits a strongly distorted perovskite structure with space group Pnma. ${ }^{8}$ The deviation of this structure from the perfect cubic perovskite structure (with space group $P m \overline{3} m$ ) can be decomposed into the following three contributions:

(i) A staggered (checkerboardlike) $Q^{x}$-type JT distortion of the oxygen octahedra within the $x-y$ plane, with the long and short axes of neighboring octahedra alternating between the $x$ and $y$ directions (see Fig. 3). This JT distortion leads to a doubling of the unit cell compared to the ideal cubic perovskite structure, with new "in-plane" lattice vectors $\vec{a}$ $=a_{0}(\hat{x}-\hat{y})$ and $\vec{b}=a_{0}(\hat{x}+\hat{y})$, where $a_{0}$ is the lattice constant of the original (undistorted) perovskite structure. Identical $x-y$ planes are stacked on top of each other along the $z$ direction. The resulting symmetry is tetragonal.

(ii) $\mathrm{GdFeO}_{3}$-type rotations (tilting) of the oxygen octahedra, leading to an additional doubling of the unit cell along the $z$ direction, with the new lattice vector $\vec{c}=2 a_{0} \hat{z}$, and a reduction to orthorhombic Pnma symmetry.

(iii) Displacements of the La cations and a deformation (strain) of the parallelepiped formed by the lattice vectors $\vec{a}$, $\vec{b}$, and $\vec{c}$, consistent with the orthorhombic crystal class.

We expect that the internal distortions of the oxygen network, i.e., components (i) and (ii) described above, have the largest effect on the $e_{g}$ bands of $\mathrm{LaMnO}_{3}$ via the ligand-field splitting, whereas the influence of the lattice strain and of the La displacements, i.e., component (iii), can be neglected. We test the validity of this hypothesis in Sec. III A.

In the following, except for the test calculations presented in Sec. III A, we therefore consider only components (i) and (ii). This means that we use the "pseudocubic" lattice vectors $\vec{a}, \vec{b}$, and $\vec{c}$ defined above, discarding any orthorhombic strain, and we place the La cations on their ideal positions, corresponding to the perfect cubic perovskite structure. For the internal distortion of the oxygen network, we use the experimental data obtained in Ref. 7, which we decompose into the pure JT distortion and the $\mathrm{GdFeO}_{3}$-type distortion, as described below. Furthermore, we use a "cubic" lattice constant $a_{0}=3.9345 \AA$, which results in the same volume per f.u. as in the experimentally observed structure. ${ }^{7}$

Table I lists the Wyckoff positions for the two inequivalent oxygen sites $\mathrm{O} 1(4 c)$ and $\mathrm{O} 2(8 d)$ in the experimentally determined Pnma structure, ${ }^{7}$ and how we decompose the corresponding structural distortion in the pure JT component (i) and the $\mathrm{GdFeO}_{3}$-type distortion (ii). The decomposition is such that $\Delta \vec{r}=\Delta \vec{r}_{\mathrm{JT}}+\Delta \vec{r}_{\mathrm{GFO}}$ and $\Delta \vec{r}_{\mathrm{JT}}$ is orthogonal to $\Delta \vec{r}_{\mathrm{GFO}}$, where $\Delta \vec{r}, \Delta \vec{r}_{\mathrm{JT}}$, and $\Delta \vec{r}_{\mathrm{GFO}}$ are the full experimental distortion and its decomposition into pure JT and $\mathrm{GdFeO}_{3}$-type distortion, respectively. Since the Wyckoff coordinates $x, y$, and $z$ can be directly interpreted as the coordinates relative to the lattice vectors $\vec{a}, \vec{b}$, and $\vec{c}$, it follows that in the purely JT distorted structure, each oxygen anion $\mathrm{O} 2$ is displaced by $\left|\Delta \vec{r}_{\mathrm{JT}}(\mathrm{O} 2)\right|=\left|\Delta x_{\mathrm{JT}}(\mathrm{O} 2) \vec{a}+\Delta y_{\mathrm{JT}}(\mathrm{O} 2) \vec{b}+\Delta z_{\mathrm{JT}}(\mathrm{O} 2) \vec{c}\right|=0.1070 \AA$. According to Eq. (6), this corresponds to a JT amplitude of $Q_{0}^{x}=0.1513 \AA=0.0385 a_{0}$.

TABLE I. Decomposition of the experimentally observed distortion of the oxygen network into JT component and $\mathrm{GdFeO}_{3}$-type (GFO) rotations. The upper four lines contain the Wyckoff positions of the two inequivalent oxygen sites $\mathrm{O} 1(4 c)$ and $\mathrm{O} 2(8 d)$ in the experimental structure (Ref. 7), in the ideal cubic perovskite structure (note that these values correspond to a quadrupled unit cell), and our decomposition in pure JT and pure $\mathrm{GdFeO}_{3}$-type components. $\Delta \vec{r}, \Delta \vec{r}_{\mathrm{JT}}$, and $\Delta \vec{r}_{\mathrm{GFO}}$ represent the corresponding full experimental distortion and its decomposition into pure JT and GFO-type distortion, respectively. $x, y$, and $z$ are the coordinates with respect to the orthorhombic lattice vectors.

\begin{tabular}{|c|c|c|c|c|c|c|}
\hline & \multicolumn{3}{|c|}{$\mathrm{O} 1(4 c)$} & \multicolumn{3}{|c|}{$\mathrm{O} 2(8 d)$} \\
\hline & $x$ & $y$ & $z$ & $x$ & $y$ & $z$ \\
\hline Expt. (Ref. 7) & -0.0733 & -0.0107 & 0.25 & 0.2257 & 0.3014 & 0.0385 \\
\hline Ideal & 0.0 & 0.0 & 0.25 & 0.25 & 0.25 & 0.0 \\
\hline $\mathrm{JT}$ & 0.0 & 0.0 & 0.25 & 0.2636 & 0.2636 & 0.0 \\
\hline GFO & -0.0733 & -0.0107 & 0.25 & 0.2122 & 0.2879 & 0.0385 \\
\hline$\Delta \vec{r}$ & -0.0733 & -0.0107 & 0.0 & -0.0243 & 0.0514 & 0.0385 \\
\hline$\Delta \vec{r}_{\mathrm{JT}}$ & 0.0 & 0.0 & 0.0 & 0.0136 & 0.0136 & 0.0 \\
\hline$\Delta \vec{r}_{\mathrm{GFO}}$ & -0.0733 & -0.0107 & 0.0 & -0.0379 & 0.0379 & 0.0385 \\
\hline
\end{tabular}



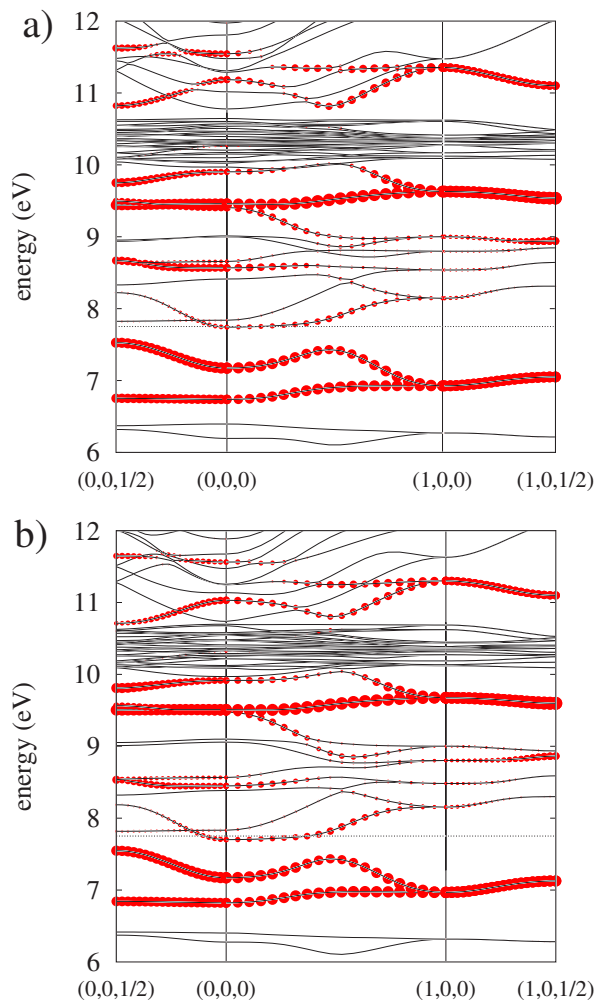

FIG. 4. (Color online) LSDA energy bands (thin lines) along high-symmetry directions of the orthorhombic Brillouin zone calculated for (a) the exact experimental structure and (b) for the simplified structure with no orthorhombic strain and the La cations on their ideal positions. Both calculations are done for $A$-type antiferromagnetic ordering. The thick dots indicate the amount of $\mathrm{Mn} e_{g}$ character in the corresponding Bloch functions. The dashed horizontal line at $\sim 7.75 \mathrm{eV}$ indicates the Fermi energy. In (b), the highsymmetry $k$ points are given in Cartesian coordinates and in units of $\pi / a_{0}$, and in (a), the corresponding $k$ points are labeled identically but correspond to the slightly strained reciprocal lattice of the experimental structure. The two cases are nearly indistinguishable.

\section{DISCUSSION OF RESULTS}

\section{A. Test of structural decomposition}

In the previous section, we stated that only components (i) and (ii), i.e., the internal distortion of the oxygen network, are important for the $e_{g}$ bands in $\mathrm{LaMnO}_{3}$ and that the lattice strain as well as the displacements of the La cations are negligible. In order to test this hypothesis, we now compare the LSDA band structure calculated for the full experimental structure of Ref. 7 with the one calculated for the slightly simplified structure described above, where the lattice strain and the La displacements are set to zero, while the internal coordinates of the oxygen anions are the same as observed experimentally.

The corresponding LSDA band structures in the energy range of the $\mathrm{Mn} e_{g}$ bands calculated for $A$-type antiferromagnetic ordering are shown in Fig. 4 along certain high symmetry directions of the orthorhombic Brillouin zone. The Mn $e_{g}$ bands are visualized by dots along the bands, with the radius of the dots proportional to the amount of $\mathrm{Mn} e_{g}$ character contained in the corresponding Bloch function. It is

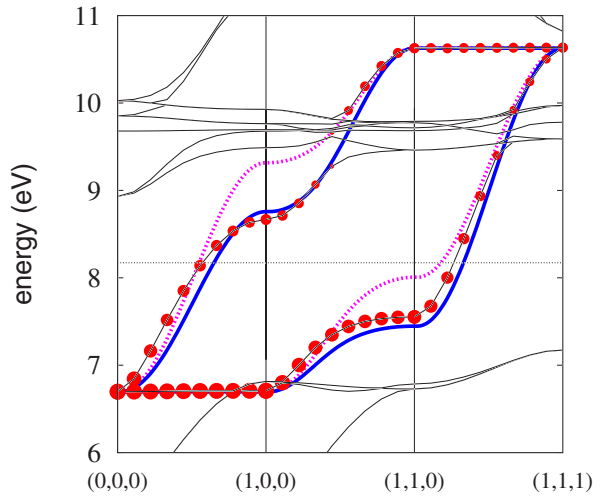

FIG. 5. (Color online) Majority-spin bands for cubic ferromagnetic $\mathrm{LaMnO}_{3}$. The LSDA band structure is represented by thin lines, with the dots indicating the amount of $\mathrm{Mn} e_{g}$ character in the corresponding Bloch function. The thick broken line is the TB fit with only nearest-neighbor hopping, whereas the thick solid line represents the TB fit including both nearest and next-nearestneighbor hopping. The thin dashed horizontal line at $\sim 8.2 \mathrm{eV}$ indicates the Fermi energy. The high-symmetry $k$ points are given in Cartesian coordinates and in units of $\pi / a_{0}$.

clearly seen that the band structures obtained for the fully experimental structure and for the simplified structure with only the oxygen distortions included are nearly indistinguishable, with only small deformations of the energy bands resulting from the orthorhombic strain and the La displacements. This validates our initial hypothesis and, in the following, we therefore analyze only the effect of the internal structural distortion of the oxygen octahedra on the dispersion of the $e_{g}$ bands.

We point out that by setting the lattice strain to zero, we also neglect any homogeneous $Q^{z}$-type JT distortion. The good agreement between the two band structures shown in Fig. 4 thus also indicates that there is no noticeable effect of $Q^{z}$ on the electronic band structure of $\mathrm{LaMnO}_{3}$ in its experimental crystal structure.

The simplified structure that gives rise to the LSDA band structure shown in the bottom part of Fig. 4 results from the superposition of distortions (i) and (ii) (described above) of the oxygen network. In the following, we will first establish the $e_{g}$ band structure of $\mathrm{LaMnO}_{3}$ in the ideal cubic perovskite structure and then separately introduce either the JT distortions, component (i), or the $\mathrm{GdFeO}_{3}$-type rotations, component (ii), and analyze the corresponding effects on the $e_{g}$ bands.

\section{B. Cubic structure}

Figure 5 shows the calculated LSDA energy dispersion around the Fermi level, calculated for ferromagnetic cubic $\mathrm{LaMnO}_{3}$ in the undistorted cubic perovskite structure with $a_{0}=3.9345 \AA$. Only the majority-spin bands are shown. Within the TB model defined in Eq. (2), Hund's coupling simply splits the spin majority and minority bands rigidly by $\Delta E=2 J$ for a ferromagnetic arrangement of the $t_{2 g}$ core spins, and we therefore discuss only the majority-spin bands in the following. The $\mathrm{Mn} e_{g}$ bands are again visualized by 
the dots along the bands in Fig. 5. It is evident that even though the Mn $e_{g}$ bands are intersected by other bands, the $e_{g}$ dispersion can be nicely traced along the dots. The $e_{g}$ bands are about half-filled, as expected from the formal electron configuration $t_{2 g}^{3} e_{g}^{1}$ of the $\mathrm{Mn}^{3+}$ ion. The bands at $\sim 7 \mathrm{eV}$ and lower, just touching the lower range of the $e_{g}$ bands, are the filled majority $\mathrm{Mn} t_{2 g}$ bands. The weakly dispersive bands slightly below $10 \mathrm{eV}$ that intersect the $\mathrm{Mn} e_{g}$ bands correspond to the La $4 f$ states, and the strongly dispersive unoccupied bands above the Mn $e_{g}$ manifold have predominantly La $d$ character.

The thick lines in Fig. 5 correspond to fits of the nearestand next-nearest-neighbor TB models for the $e_{g}$ bands. The nearest-neighbor hopping parameter $t=0.655 \mathrm{eV}$ is determined from the full $e_{g}$ bandwidth $W=3.928 \mathrm{eV}=6 t$. The next-nearest-neighbor hopping parameter $t^{\prime}$ is obtained in the following way: In the next-nearest-neighbor model, the width of the energy dispersion of the upper $e_{g}$ band between $k$ points $\Gamma=(0,0,0)$ and $X=(1,0,0)$ is equal to $\Delta E_{\Gamma X}^{(2)}=4 t$ $-16 t^{\prime}$, whereas the dispersion width of the lower band between $X$ and $M=(1,1,0)$ is equal to $\Delta E_{X M}^{(1)}=2 t-16 t^{\prime}{ }^{25}$ The corresponding energy differences obtained from the LSDA calculation are $\Delta E_{\Gamma X}^{(2)}=0.851 \mathrm{eV}$ and $\Delta E_{X M}^{(1)}=1.965 \mathrm{eV}$, leading to $t^{\prime}=0.041 \mathrm{eV}$ and $t^{\prime}=0.029 \mathrm{eV}$, respectively (and using the previously obtained $t=0.655 \mathrm{eV}$ ). An average value of $t^{\prime}=0.035 \mathrm{eV}$ is used for the TB fit in Fig. 5 and in the remaining part of this paper.

It becomes clear from Fig. 5 that the simple nearestneighbor TB model cannot reproduce the LSDA dispersion very well, whereas the next-nearest-neighbor TB model leads to a very good description of the energy dispersion for all $k$ points.

We point out that a nonmagnetic LDA calculation results in a low-spin electron configuration and the loss of the $t_{2 g}$ core spin. Indeed, if we perform a nonmagnetic LDA calculation, the $e_{g}$ bands are empty and higher in energy compared to the oxygen $p$ levels, which in accordance with Eq. (1) results in a reduced bandwidth of $3.546 \mathrm{eV}$, corresponding to a nearest neighbor hopping amplitude of $t=0.591 \mathrm{eV}$. A nonmagnetic LDA calculation is thus not necessarily a good representation of the electronic structure of the paramagnetic phase, and we therefore use the ferromagnetic state as the starting point for the model analysis. In general, this shows that an LDA+DMFT treatment of $\mathrm{LaMnO}_{3}$ based on a nonmagnetic LDA calculation, such as the one presented in Ref. 27 , leads to a slight underestimation of the electron hopping.

Next, we investigate the influence of $A$-type antiferromagnetic order. Figure 6 shows the calculated LSDA band structure for this case. Note that the underlying crystal structure is still a perfect cubic perovskite. Again, the $e_{g}$ character of the bands is visualized by the dots. The thick lines correspond to the fit within our antiferromagnetic next-nearest-neighbor TB model with the hopping parameters obtained from the ferromagnetic case. Due to the doubling of the magnetic unit cell, the number of bands is also doubled. A Hund's rule parameter $J=1.340 \mathrm{eV}$ is obtained from the energy splitting at the $\Gamma$ point between the two bands at $6.7 \mathrm{eV}$ and $9.4 \mathrm{eV}$, which show no dispersion along $\overline{\Gamma A}[A=(0,0,1 / 2)]$. This splitting is exactly equal to $2 J$ in the TB model. The value $J$

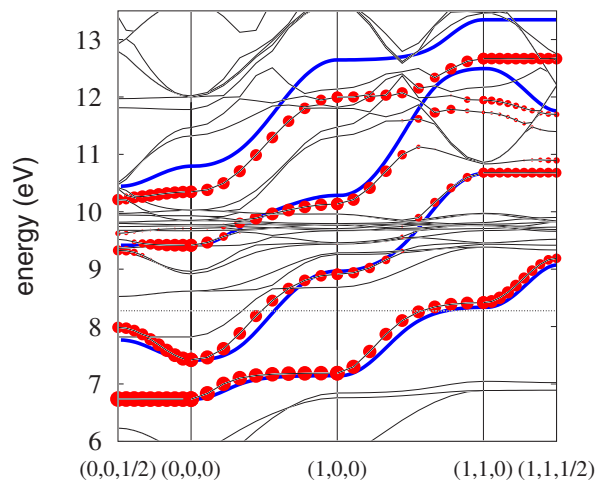

FIG. 6. (Color online) Band structure for cubic $\mathrm{LaMnO}_{3}$ with $A$-type antiferromagnetic order. The LSDA band structure is represented by thin lines, with the dots indicating the amount of Mn $e_{g}$ character in the corresponding Bloch function. The thick line represents the TB fit including both nearest and next-nearest-neighbor hoppings. The thin dashed horizontal line at $\sim 8.3 \mathrm{eV}$ indicates the Fermi energy. The high-symmetry $k$ points are given in Cartesian coordinates and in units of $\pi / a_{0}$.

$=1.340 \mathrm{eV}$ is within $2.4 \%$ of the value $J=1.309 \mathrm{eV}$ obtained from the ferromagnetic band structure as the splitting between majority- and minority-spin states at the $\Gamma$ point (not shown).

Figure 6 shows that the two lowest $e_{g}$ bands are described very well by the antiferromagnetic TB model. The upper two bands show some deviations from the model, especially in the high energy region. This is an inevitable result of the description within a pure $d$ band model. As described in Sec. $\mathrm{I}$, the "true" hopping is mediated by the oxygen $p$ orbitals and therefore the $e_{g}$ dispersion depends on the energetic distance from the oxygen $p$ levels [see Eq. (1)]. This leads to a slight overestimation of the energy dispersion for the high energy states in the pure $d$ model. The same effect can also be observed in the ferromagnetic case: Due to their higher energy relative to the oxygen $p$ states, the bandwidth of the $e_{g}$ minority-spin bands is smaller than for the corresponding majority-spin bands. The nearest-neighbor hopping parameter corresponding to the minority-spin bands in the ferromagnetic case is $t=0.548 \mathrm{eV}$. In the following, we use the value $t=0.655 \mathrm{eV}$, corresponding to the majority-spin bands in the ferromagnetic configuration, since this value is representative for the $e_{g}$ bands close to the Fermi level, which determine the important low energy behavior in manganite systems.

Figure 7 shows the dependence of both nearest and nextnearest hopping parameters on the lattice constant $a$. The nearest-neighbor hopping $t$ decreases with increasing Mn-O bond length, whereas the next-nearest-neighbor hopping $t^{\prime}$ shows a slight increase. This somewhat unexpected behavior of $t^{\prime}$ results from the fact that the energy difference between oxygen $p$ and $\mathrm{Mn} d$ states decreases with increasing volume and therefore counteracts the effect of the reduced overlap integrals for larger lattice constants.

\section{Purely Jahn-Teller distorted structure}

We now address the effect of the JT distortion of the oxygen octahedra on the $e_{g}$ bands in $\mathrm{LaMnO}_{3}$. As described 


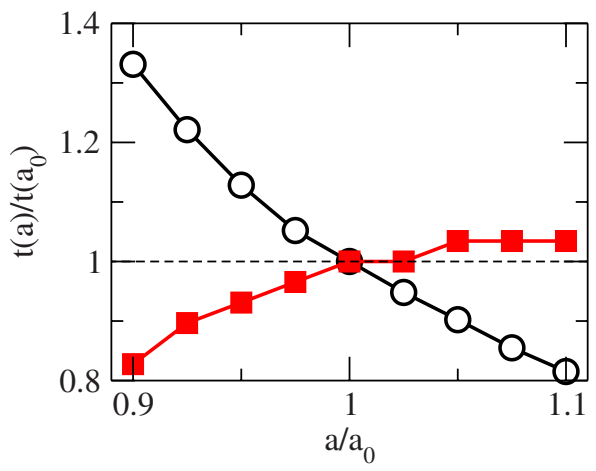

FIG. 7. (Color online) Variation of hopping amplitudes with lattice constant. Open circles correspond to the nearest-neighbor hopping $t$ and filled squares correspond to the next-nearest-neighbor hopping $t^{\prime} . a_{0}$ indicates the lattice constant derived from the experimental unit cell volume.

in Sec. II C, we consider only the internal distortions of the oxygen network. This means that we displace the oxygen anions relative to the cubic structure according to the decomposition of the Wyckoff positions described in Table I (line "JT"). This results in the staggered arrangement of JT distorted oxygen octahedra shown in Fig. 3(b), with the same JT amplitude $Q_{0}^{x}$ as in the experimental structure.

Figure 8 shows the calculated LSDA majority-spin bands for $\mathrm{LaMnO}_{3}$ in the purely JT distorted structure with ferromagnetic spin ordering. Due to the unit cell doubling within the $x-y$ plane compared to the cubic structure, the two bands corresponding to the $k$ points between $X=(1,0,0)$ and $M$ $=(1,1,0)$ in the ferromagnetic cubic case (see Fig. 5) are "backfolded" between the $k$ points $U=(1,0,0)$ and $\Gamma$ $=(0,0,0)$ in the tetragonal Brillouin zone. In addition, the two bands between $M=(1,1,0)$ and $R=(1,1,1)$ in Fig. 5 now correspond to the two upper bands between $\Gamma$

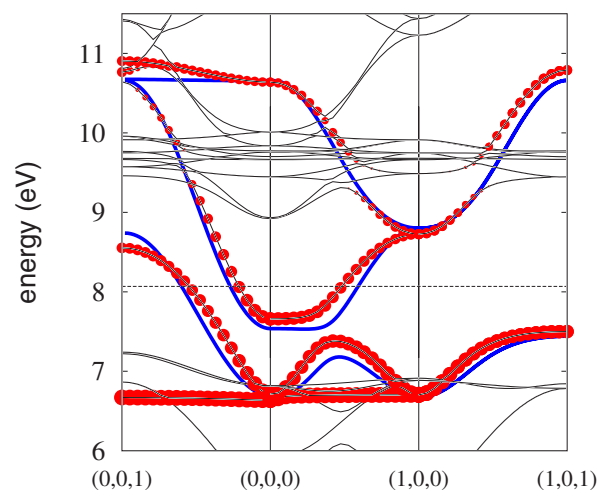

FIG. 8. (Color online) Majority-spin band structure for ferromagnetic $\mathrm{LaMnO}_{3}$ in the purely JT distorted structure [see Fig. $3(\mathrm{~b})$, where the unit cell is doubled in the $x-y$ plane. The highsymmetry $k$ points are given with respect to the Cartesian coordinate system defined by the reciprocal lattice vectors of the undistorted cubic structure. The units are $\pi / a_{0}$. The LSDA band structure is represented by thin lines with the dots indicating the amount of Mn $e_{g}$ character in the corresponding Bloch function. The thick line represents the TB fit and the thin dashed horizontal line at $\sim 8.1 \mathrm{eV}$ indicates the Fermi energy.
$=(0,0,0)$ and $Z=(0,0,1)$ in Fig. 8 , and the two bands between $U=(1,0,0)$ and $R=(1,0,1)$ in Fig. 8 are now twofold degenerate. It can be seen that the level splitting between the two original $e_{g}$ states at the $\Gamma$ point (at $\sim 6.7 \mathrm{eV}$ in Fig. 8), which are degenerate in the cubic case, is very small $(\sim 0.08 \mathrm{eV})$ and that the main effect of the JT distortion is to remove the band crossing between $\Gamma$ and $U=(1,0,0)$, resulting from the simple backfolding of the cubic band structure due to the unit cell doubling.

To obtain the value of the JT coupling constant $\lambda$ within our TB model, we first determine the $k$ point of the band crossing between $\Gamma$ and $U$ for the case of zero JT distortion. We then determine $\lambda Q_{0}^{x}$ in the model by fitting the splitting at this $k$ point to the corresponding splitting obtained from the LSDA calculation. In this way, we obtain a value of $\lambda Q_{0}^{x}$ $=0.248 \mathrm{eV}$, corresponding to $\lambda=1.639 \mathrm{eV} / \AA$, since $Q_{0}^{x}$ $=0.1513 \AA$ (see Sec. II C). Alternatively, we can also fit the small splitting of the two lowest $e_{g}$ bands at the $\Gamma$ point by numerically adjusting the JT coupling. In this way, we find $\lambda Q_{0}^{x}=0.289 \mathrm{eV}$, corresponding to $\lambda=1.910 \mathrm{eV} / \AA$, which is within $17 \%$ of the value obtained above. This shows that the extracted coupling strength does not depend critically on the fitting procedure.

The energy dispersion calculated within the TB model using the hopping amplitudes $t=0.655 \mathrm{eV}$ and $t^{\prime}=0.035 \mathrm{eV}$ obtained for the cubic structure and the JT coupling constant $\lambda=1.639 \mathrm{eV} / \AA$ obtained in the way described above is compared to the full LSDA band structure in Fig. 8. It can be seen that the dispersion of the $e_{g}$-projected bands is well described within the TB model. Some deviations occur close to the lifted band crossing between $\Gamma$ and $U=(1,0,0)$. These deviations are most likely caused by the asymmetry of the $\mathrm{Mn}-\mathrm{O}$ bonds, which is neglected in the effective $e_{g} \mathrm{~TB}$ model. The good overall quality of the TB fit shows that the TB hopping amplitudes are not affected by the presence of the JT distortion. This indicates that the model description with the assumed local coupling of the JT distortion to the $e_{g}$ levels is justified.

To test whether the linear form of the coupling term within our model is consistent with the density functional theory calculation, and to further test our procedure for obtaining $\lambda Q^{x}$ from the LSDA results, we perform additional calculations with different amplitudes of the JT distortion. The results are presented in Fig. 9, where the JT energy $\lambda Q_{0}^{x}$ is determined by fitting the band splitting between $\Gamma$ and $U$ $=(1,0,0)$. We note that $\lambda Q^{x}$ on the ordinate in Fig. 9 should be regarded as the model parameter that we obtain by our fitting procedure, whereas the abscissa $Q^{x} / Q_{0}^{x}$ characterizes the input structure for our LSDA calculation (relative to the experimentally observed JT distortion $Q_{0}^{x}$ ). It is evident that the dependence of $\lambda Q^{x}$ on the input distortion is nearly perfectly linear, which indicates the good quality of our fit and the adequacy of the linear coupling term within the model.

It becomes clear from Fig. 8 that the JT distortion in the experimentally observed structure of $\mathrm{LaMnO}_{3}$ has only a weak effect on the dispersion along $k_{z}$. This is complementary to the effect of the A-type magnetic order, which strongly suppresses the electron hopping along this direction. The insulating band structure obtained in LSDA for the fully 


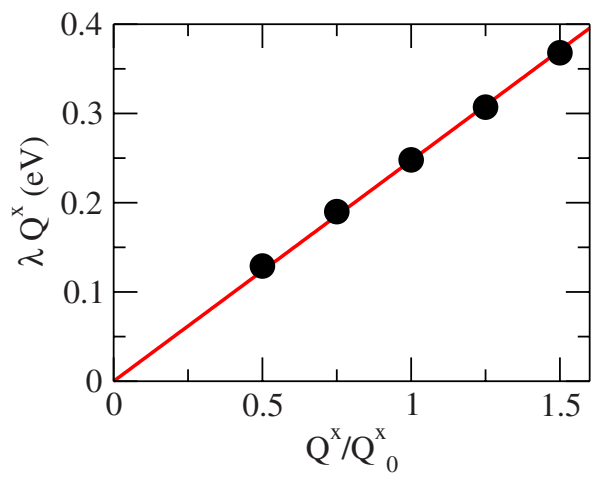

FIG. 9. (Color online) Magnitude of $\lambda Q^{x}$ obtained by fitting the TB model to LSDA calculations with different amplitudes of the JT distortion. $Q_{0}^{x}$ indicates the magnitude of the JT distortion found in the experimental structure. Filled circles are the results of the actual calculations, whereas the straight line corresponds to $\lambda Q^{x}$ $=0.247 \mathrm{eV} Q^{x} / Q_{0}^{x}$.

distorted structure with $A$-type antiferromagnetic order is therefore a combined effect of both the staggered JT distortion within the $x-y$ plane and the $A$-type antiferromagnetism. To achieve an insulating state within LSDA solely due to the JT distortion (i.e., for the ferromagnetic case) would require an unrealistically large JT amplitude. Within our TB model, a value of $\lambda Q^{x}>1.1 \mathrm{eV}$, i.e., more than four times the JT distortion of the experimental structure, is required to open up an energy gap. This is due to the large value of the hopping $t$ and the fact that for staggered JT order $H_{\mathrm{JT}}$ does not commute with $H_{\text {kin. }}$. The fact that the JT distortion alone is not enough to stabilize an insulating state in $\mathrm{LaMnO}_{3}$ has also been pointed out in Refs. 19, 21, and 28.

One possibility for explaining the insulating character of $\mathrm{LaMnO}_{3}$, as noted by previous authors, ${ }^{19}$ is that electronelectron interactions beyond LSDA increase the effective JT splitting, thereby stabilizing the insulating state. To address this, we have performed additional LSDA $+U$ calculations (which will be discussed in detail elsewhere). In the LSDA $+U$ method, the interactions between the $d$ states of the transition-metal cations are treated explicitly on the level of a mean-field Hubbard model. ${ }^{29}$ Thereby, a parameter $U$ represents the strength of the (screened) on-site Coulomb repulsion between the $d$ electrons and a parameter $\mathcal{J}^{H}$ represents Hund's coupling. In our LSDA $+U$ calculations, we use a slightly simplified approach where only $U_{\text {eff }}=U-\mathcal{J}^{H}$ enters. $^{30}$

We expect that the on-site Coulomb repulsion enhances the effect of the JT distortion and therefore drives the system toward an insulating state as the value of $U$ is increased. However, the calculated LSDA $+U$ band structure for the purely JT distorted case with $Q^{x}=Q_{0}^{x}$ and ferromagnetic spin order stays metallic even for a rather large Hubbard parameter of $U=8 \mathrm{eV}$ (see Fig. 10). The reason for this is the following: The use of the LSDA $+U$ method leads to a strong downward energy shift of the occupied part of the $e_{g}$ bands and also to a moderate overall downshift of the $d$ states as a whole. As a result of the latter, the higher-lying, mostly unoccupied $e_{g}$ bands in $\mathrm{LaMnO}_{3}$ move somewhat closer to the $\mathrm{O} 2 p$ bands, which are located in the energy range between 0

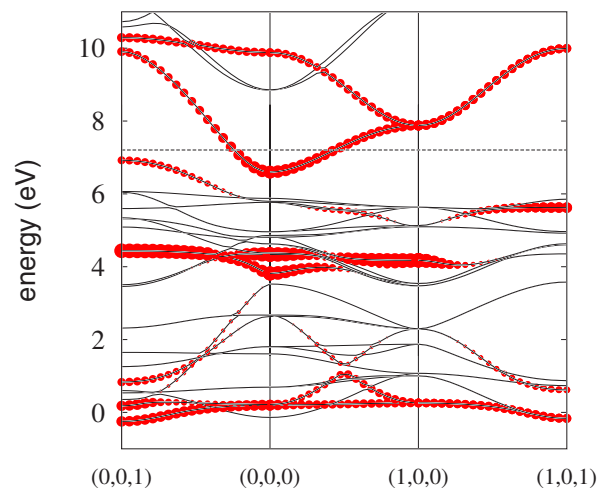

FIG. 10. (Color online) Majority-spin band structure for ferromagnetic $\mathrm{LaMnO}_{3}$ in the purely JT distorted structure calculated using the LSDA $+U$ method with $U_{\text {eff }}=7.12 \mathrm{eV}\left(U=8 \mathrm{eV}\right.$ and $\mathcal{J}^{H}$ $=0.88 \mathrm{eV})$. The high-symmetry $k$ points are given in Cartesian coordinates and in units of $\pi / a_{0}$. The Kohn-Sham band structure is represented by thin lines with the dots indicating the amount of Mn $e_{g}$ character in the corresponding Bloch function. The thin dashed horizontal line at $\sim 7.2 \mathrm{eV}$ indicates the Fermi energy. The overlapping bands in the energy range between 0 and $6 \mathrm{eV}$ have mixed O $2 p$-Mn $3 d$ character.

and $6 \mathrm{eV}$ and are not shifted within the LSDA $+U$ method. Furthermore, the strong downward shift of the occupied $d$ bands results in an energetic overlap and therefore strong hybridization between the occupied $e_{g}$ states and the $\mathrm{O} 2 p$ bands. In this case, the simple two-band TB model is not applicable any more, and some mixed $p$ - $d$ bands, which extend above the original top of the $\mathrm{O} 2 p$ states at $6 \mathrm{eV}$, reach above the Fermi level. Since the LSDA $+U$ method corrects only for the Coulomb interactions between the Mn $d$ states, it is not obvious whether this shift of the $d$ states relative to the $\mathrm{O} 2 p$ states is a real physical effect or rather an artifact of the LSDA $+U$ method (see Sec. IV for a further discussion of this point). An exact experimental determination of the energy separation between the $\mathrm{Mn} d$ and $\mathrm{O} p$ states in $\mathrm{LaMnO}_{3}$ would provide further insight on this.

\section{D. $\mathrm{GdFeO}_{3}$-type rotations}

Finally, we address the effect of the $\mathrm{GdFeO}_{3}$-type oxygen octahedra rotations. These distortions change the Mn-O-Mn bond angles and are therefore expected to alter the magnitude of the hopping amplitudes. In addition, due to the resulting symmetry lowering, this distortion will enable hopping between orbitals that was either symmetry forbidden or negligibly small in the undistorted state.

Figure 11 shows the calculated LSDA energy bands for the structure where only the $\mathrm{GdFeO}_{3}$-type distortion, component (ii) in Sec. II C, is included, whereas the JT distortion, component (i), is set to zero. The Wyckoff positions of the oxygen anions for this configuration are listed in Table I (line "GFO"). In this structure, the Mn-O-Mn bond angles are reduced from the ideal $180^{\circ}$ to about $155^{\circ}$. The thick line in Fig. 11 corresponds to a fit within the next-nearest-neighbor TB model with both nearest and next-nearest hopping amplitudes scaled by a factor of 0.7 compared to the perfectly cubic case. The high quality of the fit is striking, even though 


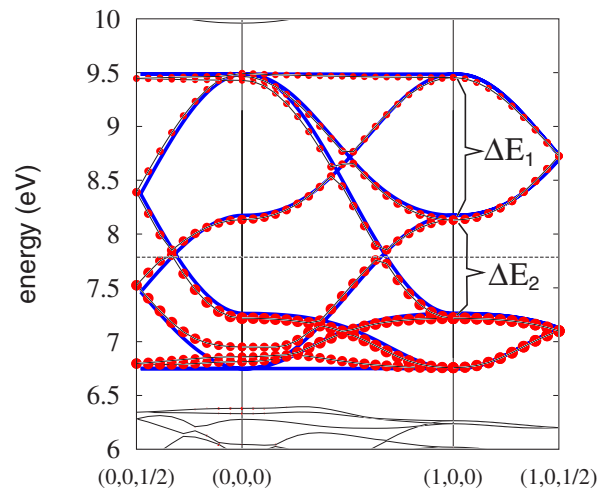

FIG. 11. (Color online) LSDA energy bands (majority spin) for ferromagnetic $\mathrm{LaMnO}_{3}$ with the experimentally observed $\mathrm{GdFeO}_{3}$-type tilting of the oxygen octahedra, but without JT distortion. The LSDA band structure is represented by thin lines with the dots indicating the amount of $\mathrm{Mn} e_{g}$ character in the corresponding Bloch function. The thick line represents the TB fit with reduced hopping amplitudes and the thin dashed horizontal line at $\sim 7.8 \mathrm{eV}$ indicates the Fermi energy. The high-symmetry $k$ points are given in Cartesian coordinates and in units of $\pi / a_{0} . \Delta E_{1}$ and $\Delta E_{2}$ mark the energy differences plotted in Fig. 12.

the LSDA band structure shows some additional dispersion at the bottom of the $e_{g}$ bands which is not accounted for in the TB model. It appears that, to a good accuracy, the oxygen tilts can be incorporated in the model simply by reducing the hopping amplitudes in an appropriate way without having to include additional hopping parameters due to the lower symmetry.

To further quantify the reduction of the hopping amplitudes as a result of the $\mathrm{GdFeO}_{3}$-type distortion, we perform calculations for different degrees of distortion, by scaling $\Delta \vec{r}_{\mathrm{GFO}}$ (see Table I) accordingly. Figure 12 shows the resulting reduction of some characteristic energy differences at the $k$ point $U=(1,0,0)$ as a function of the $\mathrm{Mn}-\mathrm{O}-\mathrm{Mn}$ bond angle. If the $\mathrm{GdFeO}_{3}$-type rotations would lead to a simple scaling of the undistorted band structure, all the lines in Fig. 12 would fall on top of each other. It can be seen that this is, in fact, a good approximation for $\mathrm{Mn}-\mathrm{O}-\mathrm{Mn}$ bond angles down to $\sim 155^{\circ}$, which corresponds to the experimentally

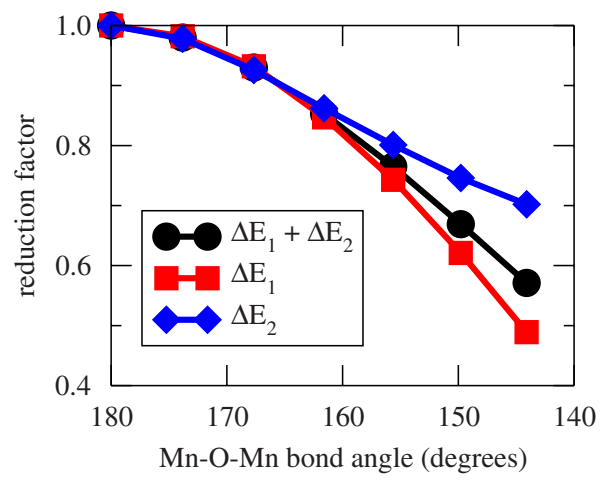

FIG. 12. (Color online) Reduction of some characteristic energy differences at the $k$ point $U=(1,0,0)$ (see Fig. 11) for different amplitudes of the $\mathrm{GdFeO}_{3}$-type distortion, which are indicated by the corresponding Mn-O-Mn bond angle. observed structure of $\mathrm{LaMnO}_{3}$. For bond angles smaller than $155^{\circ}$, the band structure starts deviating more significantly from the cubic case due to new hopping paths that become allowed in the distorted structure.

\section{SUMMARY AND CONCLUSIONS}

In summary, we have shown, by comparing LSDA bandstructure calculations to simple TB models, that the relevant electronic states in $\mathrm{LaMnO}_{3}$ are well described by a model of $e_{g}$ orbitals with nearest and next-nearest-neighbor hoppings. We have quantified the effect of changes in bond length (Fig. 7) and of the octahedral rotations (Fig. 12) on the hopping parameters, and we find that for physically relevant values (bond angles $\gtrsim 155^{\circ}$ ), the $\mathrm{GdFeO}_{3}$-type rotations significantly change the value of the hopping parameters but do not invalidate the TB description. Of particular importance is our finding that both the JT lattice distortions and the Hund's rule coupling are quantitatively accounted for by adding on-site interactions to the TB model, without changing the hopping parameters. In summary, these results justify a TB plus interaction description of manganite systems and suggest more generally that such a description is useful for transition-metal oxides.

The parameters for nearest and next-nearest-neighbor hoppings [defined in Eqs. (3) and (8)-(13)] which we obtain for the ideal cubic structure with lattice constant $a_{0}$ $=3.935 \AA$ are $t=0.655 \mathrm{eV}$ and $t^{\prime}=0.035 \mathrm{eV}$. The Hund's rule and JT coupling constants [defined in Eqs. (4) and (5)] which we obtain from our analysis are $J=1.34 \mathrm{eV}$ and $\lambda$ $=1.64 \mathrm{eV} / \AA$. Our value of $J=1.34 \mathrm{eV}$ for Hund's coupling is in excellent agreement with the exchange splitting $2 J$ $=2.7 \mathrm{eV}$ derived from $\mathrm{x}$-ray absorption measurements ${ }^{31}$ and agrees well with previous LSDA calculations. ${ }^{19,27}$ On the other hand, the strength of the JT coupling $\lambda$ obtained in this work is smaller than what has been concluded from x-ray absorption measurements in Ref. 31. It is also smaller compared to various other values obtained previously from (mostly less elaborate) fits of similar TB models to LSDA or LSDA $+U$ band-structure calculations. ${ }^{19-21,32}$

Popovic and Satpathy used a fitting procedure very similar to the one presented here and obtained a JT coupling strength of $\lambda=2.8 \mathrm{eV} / \AA$ (in our notation), compared to $\lambda$ $=1.64 \mathrm{eV} / \AA$ obtained in the present work. ${ }^{20} \mathrm{We}$ ascribe this difference to the use of the atomic sphere approximation (ASA) in the LSDA calculation of Ref. 20. In the ASA, the crystal volume is represented by an arrangement of overlapping "atomic spheres." 33 This overlap introduces an error, which furthermore depends on the amplitude of the JT distortion (since the JT distortion changes the overlap between the atomic spheres) and thus can have a pronounced effect on $\lambda$.

Ahn and Millis used a TB model very similar to the one used in this work, except that they did not include the effect of next-nearest-neighbor hopping. ${ }^{19}$ They obtained a value of $\lambda=3.38 \mathrm{eV} / \AA$ by simultaneously fitting 15 energies at four different high-symmetry $k$ points to a previous LSDA calculation for the fully distorted antiferromagnetically ordered case. It is not obvious how sensitive such a simultaneous root 
mean square fit of all the model parameters is to the exact value of $\lambda$, but we expect that the neglect of next-nearestneighbor hopping will lead to a renormalization of the other parameters of the TB model in order to account for the missing dispersion due to the next-nearest-neighbor hopping.

A value of $\lambda=2.85 \mathrm{eV} / \AA \AA$ was obtained by Yin et al. ${ }^{21}$ by calculating the dependence of several quantities on the amplitude of the JT distortion. This dependence was first obtained from LSDA $+U$ calculations within a Wannier function representation, and then compared to the corresponding results calculated for a model Hamiltonian including electron-electron interactions within the Hartree-Fock approximation. As in the case of Ref. 19 discussed above, it is not clear how sensitive this simultaneous fit of all parameters in the model Hamiltonian is to moderate changes in $\lambda$.

In contrast, the fitting procedure described in this work isolates the effect of each term in the Hamiltonian [Eq. (2)] and thus allows to obtain each parameter independently from all others. In particular, it becomes clear from our calculated band structure shown in Fig. 8 that the JT distortion does not lead to a rigid splitting of the $e_{g}$ bands, but that instead it has only subtle, albeit rather important effects on band crossings at certain $k$ points in the Brillouin zone. The reason for this is that the JT Hamiltonian $H_{\mathrm{JT}}$ in Eq. (5) for $Q^{x} \neq 0$ and staggered order does not commute with the kinetic energy term in Eq. (3) and that due to the relatively small value $\lambda Q_{0}^{x}$ $\approx 0.25 \mathrm{eV}$, the hopping energies are dominant, so that in general the effect of the JT distortion is only visible as second order shifts in the energy. In other words, at a generic $k$ point, the states picked out by the hopping term are not the eigenstates of the JT distortion. This suggests that the straightforward interpretation of peak splittings in the x-ray absorption spectra of Ref. 31 as a direct consequence of the JT distortion is not necessarily justified.

Finally, our analysis enables us to clearly identify the limitations of the effective two-band $e_{g}$ TB description of manganite systems. Our TB analysis was successful because in $\mathrm{LaMnO}_{3}$ within LSDA, the $e_{g}$ bands are well separated from the oxygen $2 p$ bands, and neither the JT distortion nor the magnetic order changes this energy spacing, and thus the value of the effective hopping, significantly. The dependence of the effective hopping parameters on the energetic distance between the $\mathrm{Mn} e_{g}$ and the $\mathrm{O} 2 p$ states [see Eq. (1)] is visible as a $15 \%-20 \%$ difference between the majority-spin and the minority-spin bandwidths and dispersion in the ferromagnetic LSDA calculation for the cubic structure (see Sec. III B) and also in the high-lying bands of the antiferromagnetic LSDA band structure shown in Fig. 6. However, in cases where the $e_{g}$ and $\mathrm{O} 2 p$ bands overlap in energy, such as, for example, in our LSDA $+U$ calculation for the purely JT distorted structure shown in Fig. 10, the effective $e_{g}$ TB analysis fails, and the $\mathrm{O} 2 p$ levels have to be taken into account explicitly.

The energy shift of the occupied $d$ states relative to the oxygen $p$ states within the LSDA $+U$ method is mainly caused by the so-called "double-counting correction," which attempts to correct for those contributions of the electronelectron interaction that are accounted for in both the LSDA and the local Hartree-Fock $(+U)$ treatment. Since the doublecounting correction is notoriously ill defined, this raises the question of whether such level shifts due to the electronelectron interaction and the resulting substantial renormalization of the effective hopping parameters are real effects, or whether this is an artifact of the LSDA $+U$ scheme, which only accounts for the static (mean-field) electron-electron interaction between the transition-metal $d$ states, while leaving the $\mathrm{O} 2 p$ states unchanged (we point out that the same problem is also present within an LDA+DMFT treatment of electronic correlations). Optical evidence (see Ref. 34) suggests that the $\mathrm{O} 2 p$ bands in manganites are located about $4 \mathrm{eV}$ below the Fermi level, consistent with the LSDA result, but more detailed investigations of the energy separation between the Mn $e_{g}$ and $\mathrm{O} 2 p$ bands will be useful for future studies.

\section{ACKNOWLEDGMENTS}

This work was supported by the MRSEC Program of the National Science Foundation under Award No. DMR0213574 (C.E.) and by the Department of Energy under Grant No. ER-46169 (A.J.M. and C.L.).
*Present address: School of Physics, Trinity College, Dublin 2, Ireland; ederer@phys.columbia.edu

${ }^{1}$ G. H. Jonker and J. H. Van Santen, Physica (Amsterdam) 16, 337 (1950).

${ }^{2}$ E. O. Wollan and W. C. Koehler, Phys. Rev. 100, 545 (1955).

${ }^{3}$ J. M. D. Coey, M. Viret, and S. von Molnár, Adv. Phys. 48, 167 (1999).

${ }^{4}$ Colossal Magnetoresistive Oxides, edited by Y. Tokura (Gordon and Breach, New York, 2000).

${ }^{5}$ E. Dagotto, T. Hotta, and A. Moreo, Phys. Rep. 344, 1 (2001).

${ }^{6}$ S. Jin, T. H. Tiefel, M. McCormack, R. A. Fastnacht, R. Ramesh, and L. H. Chen, Science 264, 413 (1994).

${ }^{7}$ P. Norby, I. G. K. Andersen, E. K. Andersen, and N. H. Andersen, J. Solid State Chem. 119, 191 (1995).

${ }^{8}$ J. B. A. A. Elemans, B. Van Laar, K. R. Van der Veen, and B. O.
Loopstra, J. Solid State Chem. 3, 238 (1971).

${ }^{9}$ J. Kanamori, J. Appl. Phys. 31, 14S (1960).

${ }^{10}$ P. M. Woodward, Acta Crystallogr., Sect. B: Struct. Sci. 53, 32 (1997).

${ }^{11}$ P. Hohenberg and W. Kohn, Phys. Rev. 136, B864 (1964).

${ }^{12}$ W. Kohn and L. J. Sham, Phys. Rev. 140, A1133 (1965).

${ }^{13}$ O. Gunnarsson, O. K. Andersen, O. Jepsen, and J. Zaanen, Phys. Rev. B 39, 1708 (1989).

${ }^{14}$ M. S. Hybertsen, M. Schlüter, and N. E. Christensen, Phys. Rev. B 39, 9028 (1989).

${ }^{15}$ W. E. Pickett and D. J. Singh, Phys. Rev. B 53, 1146 (1996).

${ }^{16}$ S. Satpathy, Z. S. Popovic, and F. R. Vukajlović, Phys. Rev. Lett. 76, 960 (1996).

${ }^{17}$ I. Solovyev, N. Hamada, and K. Terakura, Phys. Rev. Lett. 76, 4825 (1996). 
${ }^{18}$ K. Terakura, I. V. Solovyev, and H. Sawada, in Colossal Magnetoresistive Oxides, edited by Y. Tokura (Gordon and Breach, New York, 2000), pp. 119-148.

${ }^{19}$ K. H. Ahn and A. J. Millis, Phys. Rev. B 61, 13545 (2000).

${ }^{20}$ Z. Popovic and S. Satpathy, Phys. Rev. Lett. 84, 1603 (2000).

${ }^{21}$ W.-G. Yin, D. Volja, and W. Ku, Phys. Rev. Lett. 96, 116405 (2006).

${ }^{22}$ P. E. Blöchl, Phys. Rev. B 50, 17953 (1994).

${ }^{23}$ G. Kresse and J. Furthmüller, Phys. Rev. B 54, 11169 (1996).

${ }^{24}$ G. Kresse and D. Joubert, Phys. Rev. B 59, 1758 (1999).

${ }^{25}$ We use the notation of Ref. 26 to denote the high-symmetry points within cubic, tetragonal, and orthorhombic Brillouin zones.

${ }^{26}$ C. J. Bradley and A. P. Cracknell, The Mathematical Theory of Symmetry in Solids (Oxford University Press, New York, 1972).

${ }^{27}$ Y. Yamasaki, S. Miyasaka, Y. Kaneko, J.-P. He, T. Arima, and Y. Tokura, Phys. Rev. Lett. 96, 207204 (2006).
${ }^{28}$ A. Yamasaki, M. Feldbacher, Y.-F. Yang, O. K. Andersen, and K. Held, Phys. Rev. Lett. 96, 166401 (2006).

${ }^{29}$ V. I. Anisimov, F. Aryasetiawan, and A. I. Liechtenstein, J. Phys.: Condens. Matter 9, 767 (1997).

${ }^{30}$ S. L. Dudarev, G. A. Botton, S. Y. Savrasov, C. J. Humphreys, and A. P. Sutton, Phys. Rev. B 57, 1505 (1998).

${ }^{31}$ D. S. Dessau and Z.-X. Shen, in Colossal Magnetoresistive Oxides, edited by Y. Tokura (Gordon and Breach, New York, 2000), pp. 149-185.

${ }^{32}$ Note that different conventions for the JT coupling are used throughout the literature, so that care must be taken when comparing values from different sources.

${ }^{33}$ O. K. Andersen, Solid State Commun. 13, 133 (1973).

${ }^{34}$ M. Quijada, J. Černe, J. R. Simpson, H. D. Drew, K. H. Ahn, A. J. Millis, R. Shreekala, R. Ramesh, M. Rajeswari, and T. Venkatesan, Phys. Rev. B 58, 16093 (1998). 